\title{
Efficiency considerations in the construction of interpolated potential energy surfaces for the calculation of quantum observables by diffusion Monte Carlo
}

\author{
Deborah L. Crittenden \\ University of Sydney, Sydney NSW 2006, Australia \\ Keiran C. Thompson \\ School of Chemistry, University of Sydney, Sydney NSW 2006, Australia \\ Mary Chebib \\ Faculty of Pharmacy, University of Sydney, Sydney NSW 2006, Australia \\ Meredith J. T. Jordan ${ }^{\text {a) }}$ \\ School of Chemistry, University of Sydney, Sydney NSW 2006, Australia
}

School of Chemistry, University of Sydney, Sydney NSW 2006, Australia and Faculty of Pharmacy,

(Received 29 March 2004; accepted 7 April 2004)

\begin{abstract}
A modified Shepard interpolation scheme is used to construct global potential energy surfaces (PES) in order to calculate quantum observables - vibrationally averaged internal coordinates, fully anharmonic zero-point energies and nuclear radial distribution functions-for a prototypical loosely bound molecular system, the water dimer. The efficiency of PES construction is examined with respect to (a) the method used to sample configurational space, (b) the method used to choose which points to add to the PES data set, and (c) the use of either a one- or two-part weight function. The most efficient method for constructing the PES is found to require a quantum sampling regime, a combination of both $h$-weight and rms methods for choosing data points and use of the two-part weight function in the interpolation. Using this regime, the quantum diffusion Monte Carlo zero-point energy converges to the exact result within addition of 50 data points. The vibrationally averaged $\mathrm{O}-\mathrm{O}$ distance and $\mathrm{O}-\mathrm{O}$ radial distribution function, however, converge more slowly and require addition of over 500 data points. The methods presented here are expected to be applicable to both other loosely bound complexes as well as tightly bound molecular species. When combined with high quality ab initio calculations, these methods should be able to accurately characterize the PES of such species. (c) 2004 American Institute of Physics. [DOI: 10.1063/1.1756580]
\end{abstract}

\section{INTRODUCTION}

Loosely bound molecular complexes are common in chemistry. They can occur as clusters, as transition states and as reactive intermediates and are involved in many biological processes. In many cases a number of stable local minima are found on the molecular potential energy surface (PES) describing the complex. If the overall zero-point energy (ZPE) of the complex is larger than the barriers between these local minima then the ground state may be delocalized over two or more minima and the actual ground state properties may differ significantly from those of the lowest energy equilibrium configuration. For example, a delocalized proton in a hydrogen bonded complex can result in a shorter, stronger hydrogen bond, a lower proton stretching vibrational frequency and a larger nuclear magnetic resonance (NMR) chemical shift on the hydrogen bonded proton than that found in a complex where the proton is strongly localized. ${ }^{1,2}$

The degree of nuclear delocalization due to zero-point motion and tunneling may be determined by examining the

\footnotetext{
a) Author to whom correspondence should be addressed. Electronic mail: m.jordan@chem.usyd.edu.au
}

ground state nuclear wave function. This wave function may be calculated from the molecular PES using variational methods or Monte Carlo methods such as quantum diffusion Monte Carlo (DMC) ${ }^{3-6}$ Quantum diffusion Monte Carlo can be used to calculate the ground state wave function as it has the ability to determine the exact solution to the nuclear Schrödinger equation. Furthermore, Monte Carlo methods tend to have better scaling properties with increasing dimension than grid-based methods.

DMC has been used previously to study many loosely bound molecular systems or complexes. For example, the intermolecular modes in water clusters, $\left(\mathrm{H}_{2} \mathrm{O}\right)_{n}$, have been studied extensively, ${ }^{7-16}$ and some other systems studied include $(\mathrm{HF})_{2},{ }^{17}(\mathrm{DF})_{3},{ }^{18}(\mathrm{HCl})_{2},{ }^{19}(\mathrm{CO})_{2},{ }^{20} \mathrm{Ar}_{n} \mathrm{HF},{ }^{21,22}$ and $\mathrm{N}_{2}: \mathrm{H}_{2} \mathrm{O} .{ }^{23}$ These previous DMC studies have employed either functional forms for the molecular PES, for example $(\mathrm{HF})_{2},{ }^{17}\left(\mathrm{H}_{2} \mathrm{O}\right)_{n},{ }^{7-9,11-16}$ and phenol: $\left(\mathrm{H}_{2} \mathrm{O}\right)_{n},{ }^{24}$ or the direct ab initio calculation of the value of the molecular potential energy at each configuration sampled by the DMC procedure (direct DMC), for example $\left(\mathrm{H}_{2} \mathrm{O}\right)_{2} \cdot{ }^{10}$ Although using a functional form to represent the global PES is computationally inexpensive, the accuracy of the DMC calculation is limited by the inaccuracies introduced into the surface during 
parametrization. Further, this method is limited to systems for which functional forms for the PES have already been derived. Direct DMC is, in principle, the most accurate method. However, due to the high computational expense of $a b$ initio calculations and the large number of energy evaluations required per DMC simulation, this method is currently limited in accuracy by the quality of the $a b$ initio calculation performed. Typically, direct DMC simulations are only feasible using density functional theory with a double zeta quality basis. ${ }^{10}$

Interpolation between $a b$ initio data points, according to the modified Shepard interpolation scheme of Collins and co-workers, ${ }^{25-28}$ provides a method for obtaining a representation of the global PES with a higher accuracy than using a functional form and with a lower computational cost than direct DMC. The molecular PES is expressed as an interpolation over data that is scattered throughout the chemically relevant regions of conformational space. Each data point comprises a set of atomic coordinates, the corresponding energy and the first and second derivatives of the energy with respect to nuclear displacement. The potential energy at any arbitrary configuration is expressed as a weighted sum of Taylor series expansions from neighboring data points. The interpolation procedure is an iterative process in which the relevant configuration space is explored, providing a collection of molecular configurations accessed, one of which is chosen as a new data point, thus creating a new, expanded data set. This procedure is repeated until the PES is deemed to have converged, that is, when the values of the observables calculated from the PES remain constant upon addition of extra data points.

As demonstrated by Bettens, ${ }^{29}$ DMC sampling of the configuration space during this process is a cost-effective method for constructing a PES for a tightly bound system, requiring only $100 a b$ initio data points for convergence of the ground state energy of fluoromethane. The computational efficiency of this method enabled the DMC simulations to be performed on a full-dimensional composite $\operatorname{QCISD}(\mathrm{T}) / 6-311++\mathrm{G}(2 d f, 2 p)$ surface for the fluoromethane molecule and its isotopomers. However, extension of this method to loosely bound systems is expected to pose a more difficult computational task, as the volume of configuration space that needs to be covered by the interpolated PES is much larger. Therefore, it is necessary to optimize the interpolation procedure to most efficiently construct surfaces for loosely bound complexes.

In this paper, we aim to determine the most efficient methods for generating molecular potential energy surfaces for DMC calculation of the ground state energy and vibrationally averaged molecular properties of loosely bound systems. In particular, we examine the effects of using different regimes both to sample configuration space and to choose which points to add to the PES data set. We also investigate the effects of using a one- or two-part weight function in the interpolation.

The water dimer has been chosen as a test system, as it is a prototypical loosely bound system for which analytic representations of the PES have already been derived. ${ }^{30-33}$ The advantages of an analytic functional form for the PES are twofold. First, the exact ground state energy and properties can be calculated by performing DMC calculations directly on the analytic surface. Second, the calculation of the energies, first and second derivatives required for the modified Shepard interpolation is trivial. The computational effort required for DMC calculations on the interpolated potential energy surfaces is therefore determined only by the DMC convergence properties, allowing different possible regimes in the interpolation to be examined with relatively little computational expense. Our aim is, in future work, to use the most efficient of these PES-building regimes together with high quality $a b$ initio calculations, to develop accurate PES for similar loosely bound complexes.

\section{COMPUTATIONAL METHODS}

\section{Molecular potential energy surface}

The water dimer potential of Reimers, Watts, and Klein, ${ }^{32}$ as reported by Suhm and Watts, ${ }^{8}$ has been used in this work and we denote this potential as SW91. This potential is a version of the Watts semiempirical model for water. ${ }^{34}$ The SW91 potential uses slightly different parameters to the RWK2 water dimer potential reported in an earlier paper by Coker and Watts. ${ }^{16}$ The ZPE of the SW91 potential is $1.4 \%$ lower than that of the RWK2 potential ${ }^{8}$ and the RWK2 potential has been more widely used in the literature., ${ }^{9,14,16,35}$ The purpose of this work, however, has been to determine the most efficient methods for characterizing interpolated PES for loosely bound systems, and the SW91 PES is suitable for this purpose.

The SW91 PES has been used in lieu of ab initio calculations to provide the data points required for the modified Shepard interpolation. Details of the PES interpolation and iteration techniques may be found elsewhere ${ }^{25-28}$ but are briefly described below. Each PES data point $i$ comprises a set of atomic coordinates, $\mathbf{Z}_{i}$, the corresponding potential energy, $V\left(\mathbf{Z}_{i}\right)$ and the first, $f^{\prime}\left(\mathbf{Z}_{i}\right)$, and second, $f^{\prime \prime}\left(\mathbf{Z}_{i}\right)$, derivatives of the potential energy with respect to nuclear displacement. The coordinates $\mathbf{Z}_{i}$ are linear combinations of inverse bond length coordinates and have been found previously to be an efficient means of representing the asymptotic regions of the PES. ${ }^{25}$

An estimate of the potential energy at any arbitrary configuration $\mathbf{Z}$, not included in the original data set, can be given by a Taylor series expansion, $T_{i}(\mathbf{Z})$, about any of the data points, $\mathbf{Z}_{i}$, that is

$$
T_{i}(Z)=V\left(Z_{i}\right)+f^{\prime}\left(Z_{i}\right)\left(Z-Z_{i}\right)+\frac{1}{2} f^{\prime \prime}\left(Z_{i}\right)\left(Z-Z_{i}\right)^{2}+\cdots .
$$

The Taylor series expansion becomes more accurate as higher order terms are included and as the arbitrary configuration gets closer to an existing data point. The closest data points should therefore play the largest role in determining the energy at $\mathbf{Z}$ and this is achieved by weighting the Taylor expansions about each existing data point, $\mathbf{Z}_{i}$, according to the distance between $\mathbf{Z}$ and $\mathbf{Z}_{i}$. The potential energy at $\mathbf{Z}$, $V(\mathbf{Z})$, is then given as the weighted sum of Taylor expansions about of the $N$ existing data points, 


$$
V(Z)=\sum_{g \in G} \sum_{i=1}^{N} w_{g \circ i}(Z) T_{g \circ i}(Z) .
$$

The weight function, $w_{i}(z)$, is normalized by construction,

$$
w_{i}(\mathrm{Z})=\frac{v_{i}(Z)}{\sum_{g \in G} \sum_{i=1}^{N} v_{g \circ i}(Z)},
$$

where $v_{i}$ represents the "primitive" weight function. Two approaches have been used to the primitive weight function. The first is to use a simple one-part weight function,

$$
v_{i}(Z)=\left\|Z-Z_{i}\right\|^{-p}
$$

with $p>3 N-3$ chosen sufficiently large to guarantee convergence of the PES. ${ }^{25}$ Here we use $p=24$. This form of the weight function is simple to implement and computationally fast, considerations that may be important when large-scale DMC simulations are undertaken. The second form of the weight function considered is a two-part weight function including terms derived from a Bayesian analysis of the interpolation data, ${ }^{27,28}$

$$
\begin{aligned}
v_{i}(Z)= & \left\{\left[\sum_{k=1}^{N(N-1) / 2}\left(\frac{Z_{k}-Z_{k}^{(i)}}{d_{k}{ }^{(i)}}\right)^{2}\right]^{q}\right. \\
& \left.+\left[\sum_{k=1}^{N(N-1) / 2}\left(\frac{Z_{k}-Z_{k}^{(i)}}{d_{k}{ }^{(i)}}\right)^{2}\right]^{p}\right\}^{-1},
\end{aligned}
$$

where $d_{k}(i)$ represents a confidence length and $p>3 N-3$, $q \geqslant 2$, and $q \ll p$. Here we have used $p=24$ and $q=2$. The confidence lengths were derived from the distance between each data point and its closest 50 neighboring data points. This type of weight function is more complex than a simple one-part weight function but has been shown to give significantly greater accuracy in reactive systems. ${ }^{26,27}$ The water dimer, however, is a bound system and we investigate the effects of a one- or two-part weight function on modelling its PES.

The Taylor series expansion, $T_{i}(\mathbf{Z})$, in Eq. (2) has been truncated at second order, which has been previously shown to provide the most efficient means of generating a reactive PES. ${ }^{36}$ The sums in Eqs. (2) and (3) are taken over $N \times G$ configurations, where $N$ is the number of unique data points and $G$ is the order of the permutation symmetry group for the system. In this way the invariance of the PES to permutation of identical nuclei is explicitly included. For the water dimer, the full $(G=48)$ nuclear permutation symmetry has been included.

Initially, the interpolated PES was described by a single data point: the configuration at the global minimum of the SW91 PES. This configuration possesses $C_{s}$ symmetry and its structure is illustrated in Fig. 1.

\section{Quantum diffusion Monte Carlo}

The initiation phase of the DMC simulation involved establishing a population of $M$ replicas and guessing a trial ground state energy $\left(E_{T}\right)$. Each replica was then allowed to diffuse and branch for $n_{\text {step }}$ time steps each of length $\tau$, after which the trial energy was updated. This procedure was re-
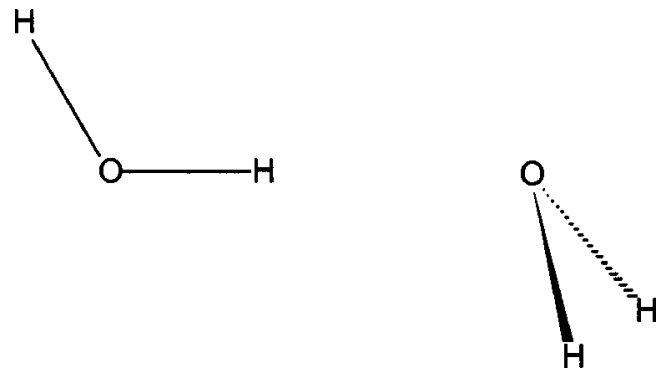

FIG. 1. Schematic representation of the global minimum energy configuration of the water dimer on the SW91 potential energy surface.

peated for $n_{\text {eq }}$ blocks, until the population had reached a steady state and the trial energy remained approximately constant. The simulation was then run for further $n$ blocks, during which time the ground state energy and wave function histogram were accumulated.

The initial ensemble of replicas for the DMC simulations was established by allowing a random fraction of a $0.5 \mathrm{bohr}$ displacement along each Cartesian axis of each atom from the equilibrium geometry. The reference potential energy $\left(V_{\text {old }}\right)$ of the ensemble was then calculated. For each time step, the atomic displacements were sampled from a Gaussian distribution with variance,

$$
\operatorname{var}=\frac{2 \tau}{\operatorname{mass}},
$$

where mass is the mass, in atomic units, of each atom considered.

The new potential energy ( $\left.V_{\text {curr }}\right)$ of the ensemble was evaluated before the branching step. During the branching step, the branching weight of each replica,

$$
w_{i}=e^{-\tau\left(0.5\left(V_{\text {old }}+V_{\text {curr }}\right)-E_{T}\right)} e^{\text {rand }}
$$

was used to determine the number of replicas created, where rand is a random number between zero and one. For weights lower than 1.0, the replica was annihilated. For replicas with higher weights, $w_{i}-1$ extra replicas were created, to give total population $P_{\text {curr }}$.

After $n_{\text {step }}$ time steps, the trial energy was updated, according to

$$
E_{T}=E_{T}+\frac{\sum_{n_{\text {step }} \frac{1}{\tau}} \log \left(\frac{P_{\text {old }}}{P_{\text {curr }}}\right)}{n_{\text {step }}} .
$$

After $n_{\text {eq }}$ equilibration blocks, the ground state energy was accumulated by averaging $V_{\text {curr }}$ and the wave function histogram was accumulated by binning the replicas for the remaining $n$ blocks.

All the results presented in this paper were obtained with the following parameters: $M=1000, \tau=0.1$ a.u., $n_{\text {step }}=300$, $n_{\mathrm{eq}}=60, n=300$, and $E_{T}($ initial $)=0.1 E_{h}$, starting with a configuration at the global minimum of the SW91 PES. The sampling of configuration space for iteratively improving the PES was carried out with identical DMC parameters, with the exception of $n$, which was decreased to 200 and $n_{\text {step }}$, which was decreased to 100 . 
The DMC simulations were repeated $N=10$ times. The results below are quoted with errors corresponding to twice the standard error of the mean, $2 \sigma / \sqrt{ } N$, where $\sigma$ is the standard deviation of the 10 runs.

Vibrationally averaged internal coordinates were calculated for each DMC simulation run, averaging over 300 blocks. Standard errors were determined as above.

\section{Iteratively improving the PES}

The interpolated PES was improved by adding data points to the initial minimum energy configuration. Both DMC and classical trajectory calculations were used to provide data points for the PES. Classical trajectories were used as a comparison for DMC because classical trajectory sampling had been used previously to develop a number of interpolated PESs for use in both quantum ${ }^{37-40}$ and classical $^{25-28,36,41-48}$ reaction dynamics. A single DMC sampling regime was used previously by Bettens. ${ }^{29}$ Both sampling methods explore the regions of configuration space important to the ground state wave function.

Configurations sampled by DMC or classical trajectory simulation were added to the PES data set according to a number of regimes with each regime yielding a different final PES. For regimes (A)-(D) described below, a DMC simulation was run using the parameters specified above. Configurations accessed during the DMC simulation were printed to file once every 10 blocks. These configurations were then sampled and the chosen points were added to the PES data set.

For regime (E), 1000 classical trajectories were calculated, using a velocity-Verlet algorithm with a time step of 0.001 a.u. The classical trajectories were propagated for 10000 time steps and the trajectory configurations were saved every 50 time steps and then sampled to provide further data points for the PES data set. The initial conditions for the trajectories were determined by microcanonical sampling, for a given initial energy. The PES was built up by adding 50 data points from trajectories run at a number of initial energies, starting from $0.005 E_{h}$ above the minimum of the SW91 PES and incrementing the energy by $0.005 E_{h}$. This process was repeated 10 times, resulting in the addition of 500 data points with a total maximum initial energy of $0.05 E_{h}$.

The two methods that have previously been used to choose which points to add from the sampled configuration space are as follows:

(1) $h$-weight sampling. ${ }^{25}$ Using this method the $n$ configurations with the largest $h$-weight, Eq. (9), were added to the PES data set for the next iteration,

$$
h[Z(j)]=\frac{1}{N-1} \frac{\sum_{n=1, n \neq j}^{N} v_{j}[Z(n)]}{\sum_{g \in G} \sum_{i=1}^{N} v_{g_{\circ} i}[Z(j)]} .
$$

rms sampling. ${ }^{28,49}$ The single sampled configuration with the largest variance in predicted energy, $\sigma_{v}$ as calculated in Eq. (10), was added to the PES data set,

$$
\sigma_{v}^{2}(Z)=\sum_{g \in G} \sum_{i=1}^{N} w_{g \circ i}(Z)\left[T_{g \circ i}(Z)-V\right]^{2} .
$$

That is, the configuration $\mathbf{Z}$ for which the Taylor series estimates of the energy, $T(\mathbf{Z})$, from the different existing data points, $\mathbf{Z}_{i}$, were most variant, was chosen for addition to the PES data set. This method, however, allows only a single configuration to be added to the data set in any iteration.

These sampling methods may be utilized alone or in combination. The potential energy surfaces were constructed using the one-part weight function, given above in Eqs. (3) and (4), or the two-part weight function, Eq. (5). The particular sampling regimes considered were the following.

(a) 1rms1hwt: add one DMC-sampled rms configuration and one DMC-sampled $h$-weight configuration to the PES data set per iteration.

(b) 1rms9hwt: add one DMC-sampled rms configuration and nine DMC-sampled $h$-weight configurations to the PES data set per iteration.

(c) allrms: add one DMC-sampled rms-sampled configuration to the PES data set per iteration.

(d) allhwt: add one DMC-sampled $h$-weight configuration to the PES data set per iteration.

(e) trajectory: alternatively add either one trajectorysampled rms configuration or one trajectory-sampled $h$-weight configuration to the PES data set per iteration. This combination of addition of one rms-sampled configuration and one $h$-weight sampled configuration has previously been demonstrated to be the most efficient method for constructing a trajectory-sampled PES. ${ }^{28}$

The predicted energies of data points added to the PES data set from the classical trajectory sampling were restricted to the amount of energy available to the system, according to the trajectory initial conditions. The maximum total energy considered was $0.05 E_{h}$, approximated $0.015 E_{h}$ above the ZPE. The DMC simulations, however, sampled configurations significantly higher in energy than the ZPE. These configurations only occurred with low probability and did not contribute significantly to the overall averaged ZPE but were weighted highly by the two sampling schemes considered. In an attempt to prevent the interpolated PES from being dominated by such configurations, DMC-sampled configurations with a predicted energy above $1.0 E_{h}$ were not included in the PES data set. If necessary, the choosing procedure was repeated until an eligible point was added to the PES data set.

The potential energy, gradients and second derivatives were calculated on the SW91 PES at each configuration added to the PES data set. Convergence of the interpolated PESs with respect to the ZPE was checked by performing 10 full DMC simulation runs on potential energy surfaces comprising $1,10,25,50,75$, and 100 data points. Initially, these convergence runs were performed using an additional $1.0 E_{h}$ screening cutoff in the actual energy, in analogy to the conditions under which the PES was grown. That is, data points with an actual energy above $1.0 E_{h}$ were not included in the PES data set. The effect of high energy points on the topol- 
TABLE I. Water dimer zero-point energy $(\mathrm{kJ} / \mathrm{mol})$ for the various PES iteration regimes considered using a one-part weight function in the PES interpolation.,

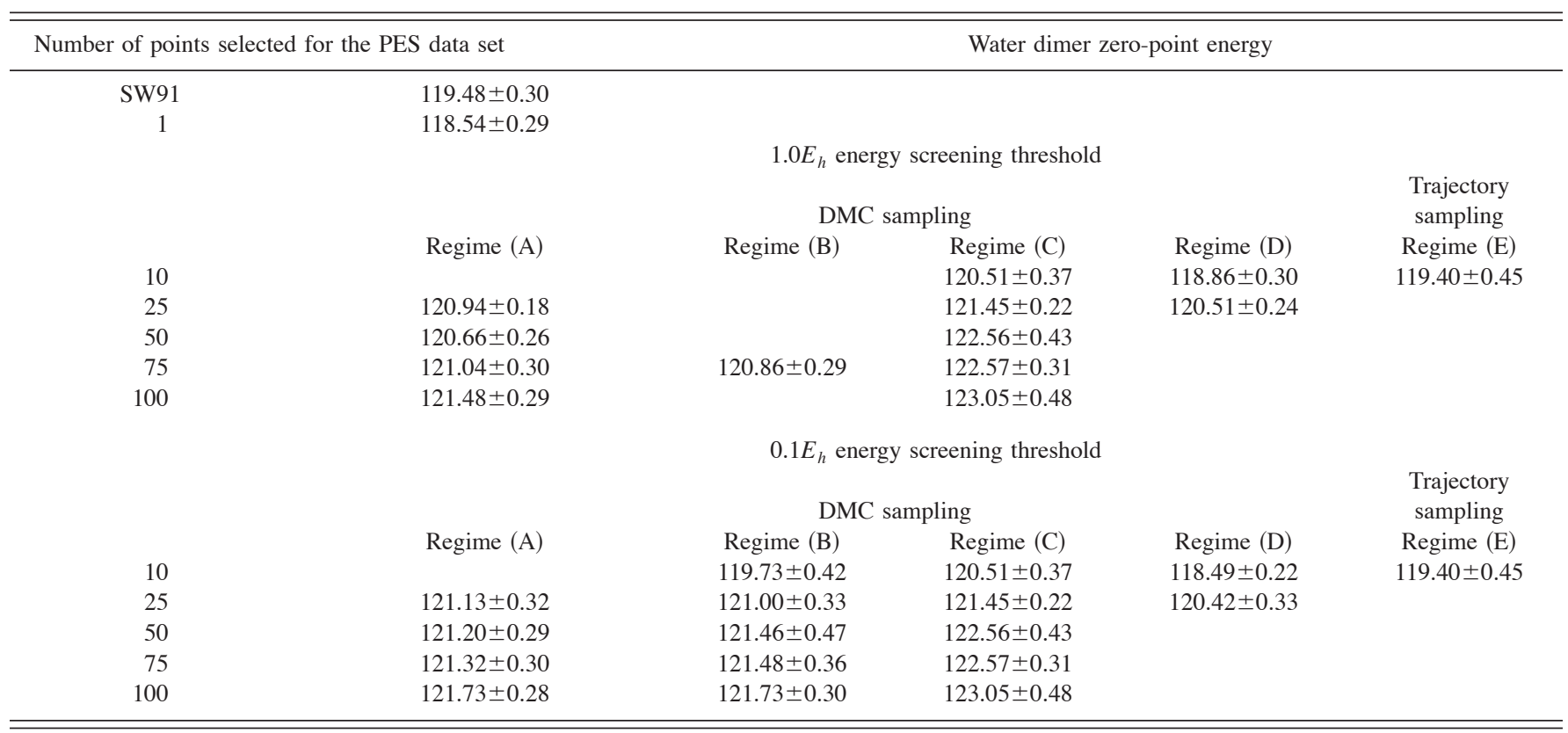

aero-point energy (ZPE) represents ZPE, as calculated on the given PES $+25.52 \mathrm{~kJ} / \mathrm{mol}$ dimer association energy, uncertainties represent two standard errors of the mean, as calculated from 10 independent DMC simulations.

${ }^{\mathrm{b}}$ Where the PES interpolation scheme resulted in negative ZPE, the table entry has been omitted.

ogy of the PES was further examined by decreasing the screening cutoff to $0.1 E_{h}$ and repeating the convergence runs. These convergence runs were performed using both the one-part and two-part weight functions. 10 reference DMC simulations were also performed on the analytic SW91 PES to provide the exact ground state energy and vibrationally averaged internal coordinates, as a measure of the convergence of the various interpolated surfaces.

Once the most efficient method for constructing the PES had been determined, the surface was then grown 500 data points using this method. Convergence of the PES with respect to the nuclear vibrational wave function was then checked by performing an additional 10 full DMC simulation runs on potential energy surfaces comprising 200, 300, 400 , and 500 data points. These convergence runs were performed using the two-part weight function only, with a $0.1 E_{h}$ screening cutoff in the actual energy of the PES data points. To enable comparison of the efficiency of this scheme with other PES growing schemes in the literature, ${ }^{25-28,36-48}$ the regime (E) surface was also grown to 500 data points, with convergence checks run every 100 data points.

Calculations were performed using computational resources at the School of Chemistry, University of Sydney and at the Australian Partnership for Advanced Computing (APAC) National Facility based at the Australian National University.

\section{RESULTS AND DISCUSSION}

\section{Zero-point energy}

The value of the ZPE calculated on the analytic SW91 surface was found to be $93.96 \pm 0.30 \mathrm{~kJ} / \mathrm{mol}$, where the uncertainty represents two standard errors of the mean. Includ- ing a correction term of $25.52 \mathrm{~kJ} / \mathrm{mol}$, corresponding to the dimer association energy, yielded a ZPE of $119.48 \pm 0.30 \mathrm{~kJ} /$ mol, in agreement with previous work. ${ }^{8}$ The ZPE on the interpolated PES described by the minimum energy configuration alone was significantly lower, $118.54 \pm 0.29 \mathrm{~kJ} / \mathrm{mol}$.

Initially, convergence runs to determine the $\mathrm{ZPE}$ were carried out on potential energy surfaces obtained using the five sampling methods, with a one-part weight function and an energy screening threshold of $1.0 E_{h}$ : data points with an actual energy greater than $1.0 E_{h}$ were not included in the PES data set. In actuality, this threshold energy did not exclude any of the data points initially selected for the various PES. The results from these convergence runs are reported in Table I. A number of the entries in this table have been omitted, as the ZPEs obtained were unphysically low as an artifact of the interpolation scheme. Data points added at relatively high energies possess large gradients that can contribute to the prediction of unphysically low or negative energies at nearby configurations. Effectively, this produced unphysical "holes" in the PES which, when encountered during a DMC simulation, resulted in the wave function becoming localized with a concomitant lowering of the calculated ZPE. In an attempt to exclude the possibility of hole formation, a second set of convergence runs were carried out also using the one-part weight function but screening out data points with an actual energy above $0.1 E_{h}$. This screening threshold resulted in $34,6,32$, and 0 points being excluded from the 100 point PES data sets for regimes (A), (B), (C), and (D), respectively. The lower screening energy threshold did not affect the trajectory-sampled surfaces. The water dimer ZPE using this lower energy threshold is also reported in Table I. The $0.1 E_{h}$ screening threshold resulted in some improvement in the quality of the potential energy sur- 
TABLE II. Water dimer zero-point energy $(\mathrm{kJ} / \mathrm{mol})$ for the various PES iteration regimes considered using a two-part weight function in the PES

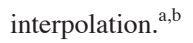

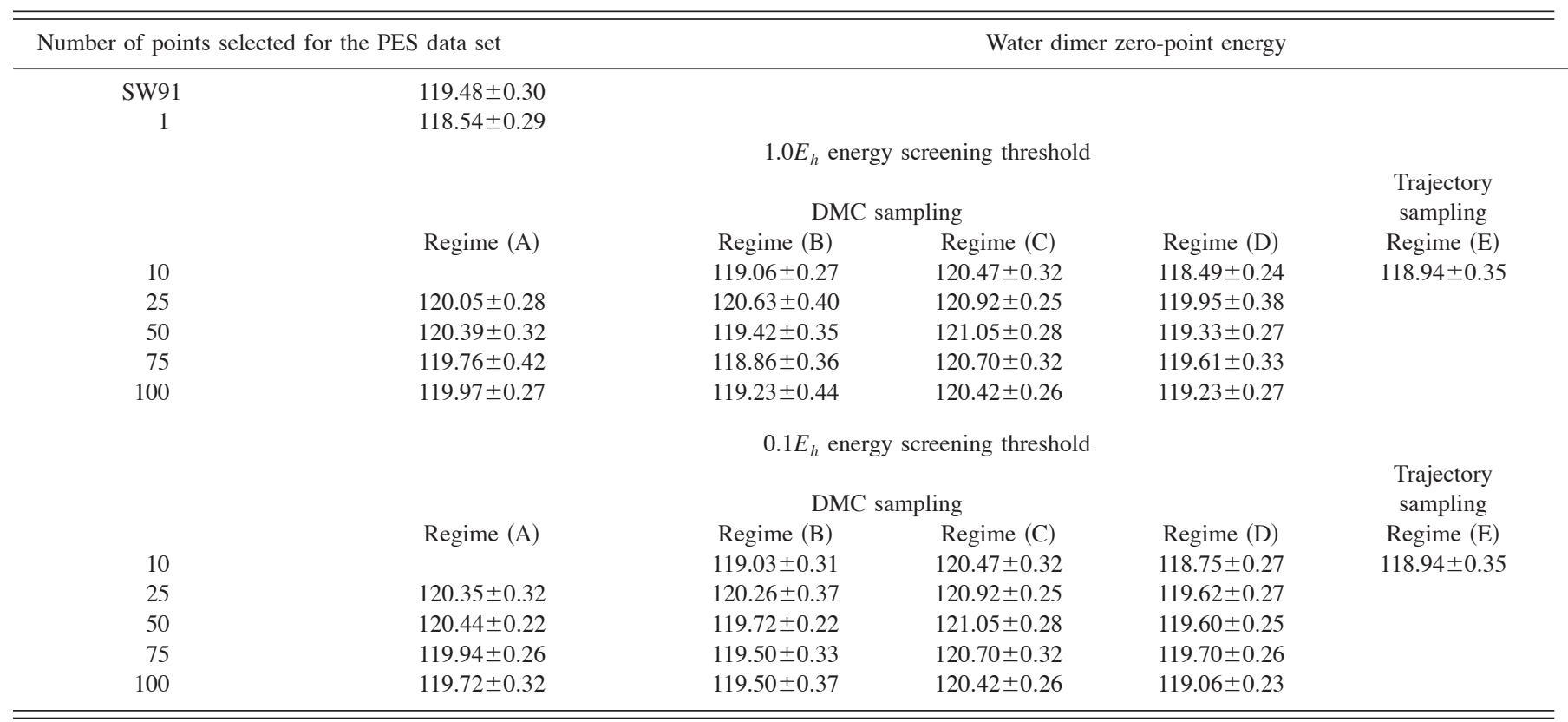

aero-point energy (ZPE) represents ZPE, as calculated on the given PES $+25.52 \mathrm{~kJ} / \mathrm{mol}$ dimer association energy, uncertainties represent two standard errors of the mean, as calculated from 10 independent DMC simulations.

${ }^{\mathrm{b}}$ Where the PES interpolation scheme resulted in negative ZPE, the table entry has been omitted.

faces obtained, with a lower incidence of holes appearing in the PES's. However, in general, these one-part weight function potential energy surfaces demonstrated poor convergence with respect to the $\mathrm{ZPE}$, appearing to converge to a value approximately $2.5 \mathrm{~kJ} / \mathrm{mol}$ higher than the ZPE for analytic SW91 surface.

The trajectory-sampled surface [regime (E)] possessed spurious wells, which were introduced after the addition of 10 data points but not yet corrected upon addition of 100 data points. This is a result of the classical trajectories spending a disproportionate amount of time in the high energy regions of the PES, corresponding to the classical turning points. Hence, more data points are added in these regions of the PES and the process of correcting holes introduced into the surface is inefficient as low energy regions of the PES are under-sampled. However, as the number of data points in- creases, the PES must converge to the exact surface, and the ZPE must converge to the analytic result.

The implication of these results for regimes $(A)-(E)$ is that convergence of potential energy surfaces described using the one-part weight function is relatively slow.

A further set of convergence runs were carried out on the surfaces described using the two-part weight function, which is known to give a more accurate description of reactive PES. ${ }^{27,28}$ These convergence runs were also carried out using energy screening thresholds of 1.0 and $0.1 E_{h}$. The results are reported in Table II and illustrated in Figs. 2 and 3 for the 1.0 and $0.1 E_{h}$ screening thresholds, respectively. These potential energy surfaces appear to converge to the exact ZPE for the DMC-sampling regimes $[(\mathrm{A})-(\mathrm{D})]$. Again, the trajectory-sampling regime $(\mathrm{E})$ yielded negative zero-point energies, as a consequence of spurious holes introduced into

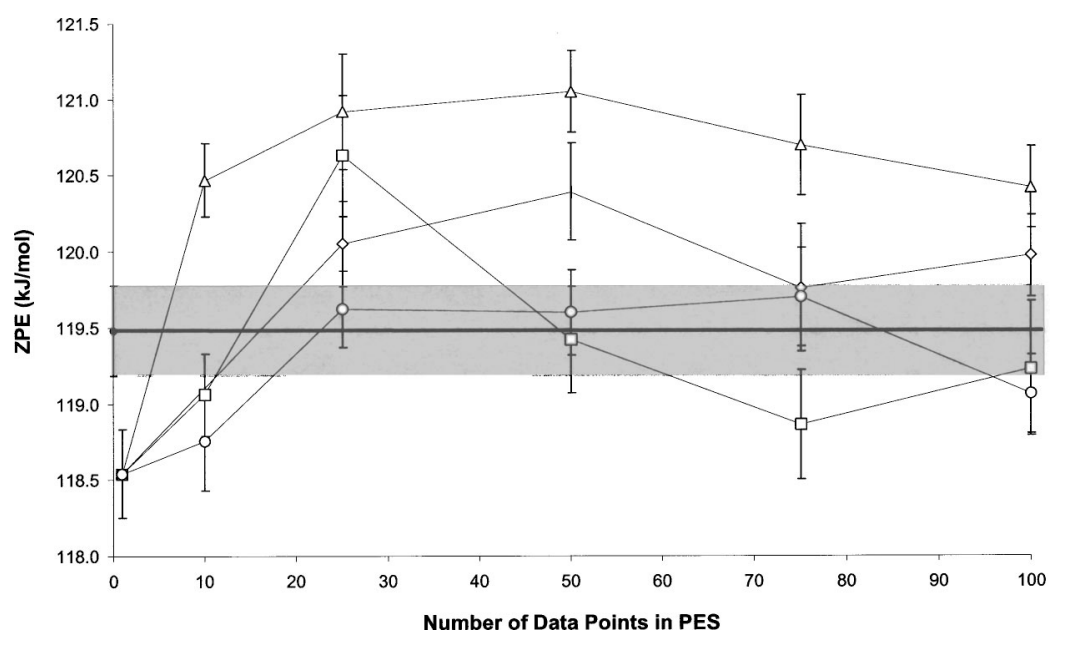

FIG. 2. Convergence, with number of unique data points describing the PES, of the $\left(\mathrm{H}_{2} \mathrm{O}\right)_{2} \mathrm{ZPE}$, as calculated in Table II, for the various two-part weight interpolated PESs calculated using a $1.0 E_{h}$ energy screening threshold. The DMC sampling regimes are indicated with open symbols: regime $(\mathrm{A})(\diamond)$, regime (B) $(\square)$, regime $(C)(\bigcirc)$, and regime $(D)(\triangle)$. For reference, the exact result on the SW91 PES is indicated at zero data points with a closed circle, with its error indicated by the shaded rectangle. All errors represent two standard errors of the mean as calculated from 10 independent DMC simulations. 


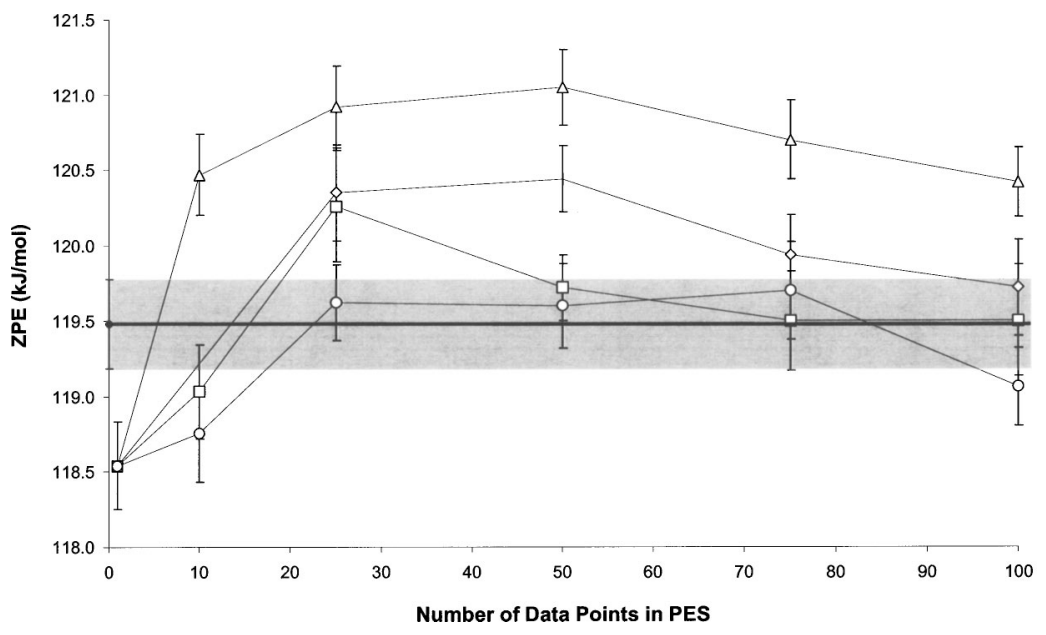

FIG. 3. Convergence, with number of unique data points describing the PES, of the $\left(\mathrm{H}_{2} \mathrm{O}\right)_{2} \mathrm{ZPE}$, as calculated in Table II, for the various two-part weight interpolated PESs calculated using a $0.1 E_{h}$ energy screening threshold. The DMC sampling regimes are indicated with open symbols: regime $(\mathrm{A})(\diamond)$, regime (B) $(\square)$, regime $(C)(\bigcirc)$, and regime (D) $(\triangle)$. For reference, the exact result on the SW91 PES is indicated at zero data points with a closed circle, with its error indicated by the shaded rectangle. All errors represent two standard errors of the mean as calculated from 10 independent DMC simulations.

the interpolated PES. Although incrementally "building up" the PES was hoped to provide adequate sampling of the lowenergy regions of the PES, this method proved unsuccessful with both weight functions considered.

The use of 1.0 or $0.1 E_{h}$ data point energy screening cutoffs appeared to have little effect on the calculated zeropoint energies, indicating that the two-part weight function has the capacity to smoothly interpolate high energy data points. The potential energy profiles along the $\mathrm{O}-\mathrm{O}$ stretching normal mode displacement vector for the regime (A), (B), (C), (D), and (E) surfaces after addition of 100 data points were generated using both 0.1 and $1.0 E_{h}$ energy screening cutoffs (Fig. 4). Inspection of these PES profiles reveals that the $0.1 E_{h}$ cutoff significantly improved the convergence of the regime $(\mathrm{C})$ profile in this degree of freedom, and slightly enhanced convergence of the regime (A) and (B) profiles.

Regimes (A), (B), and (C) all appear to converge the water dimer ZPE upon the addition of 25-50 unique data points to the PES data set when a two-part weight function is used in the PES interpolation. The most computationally efficient of these regimes is regime $(\mathrm{B})$, the addition of $1 \mathrm{rms}$
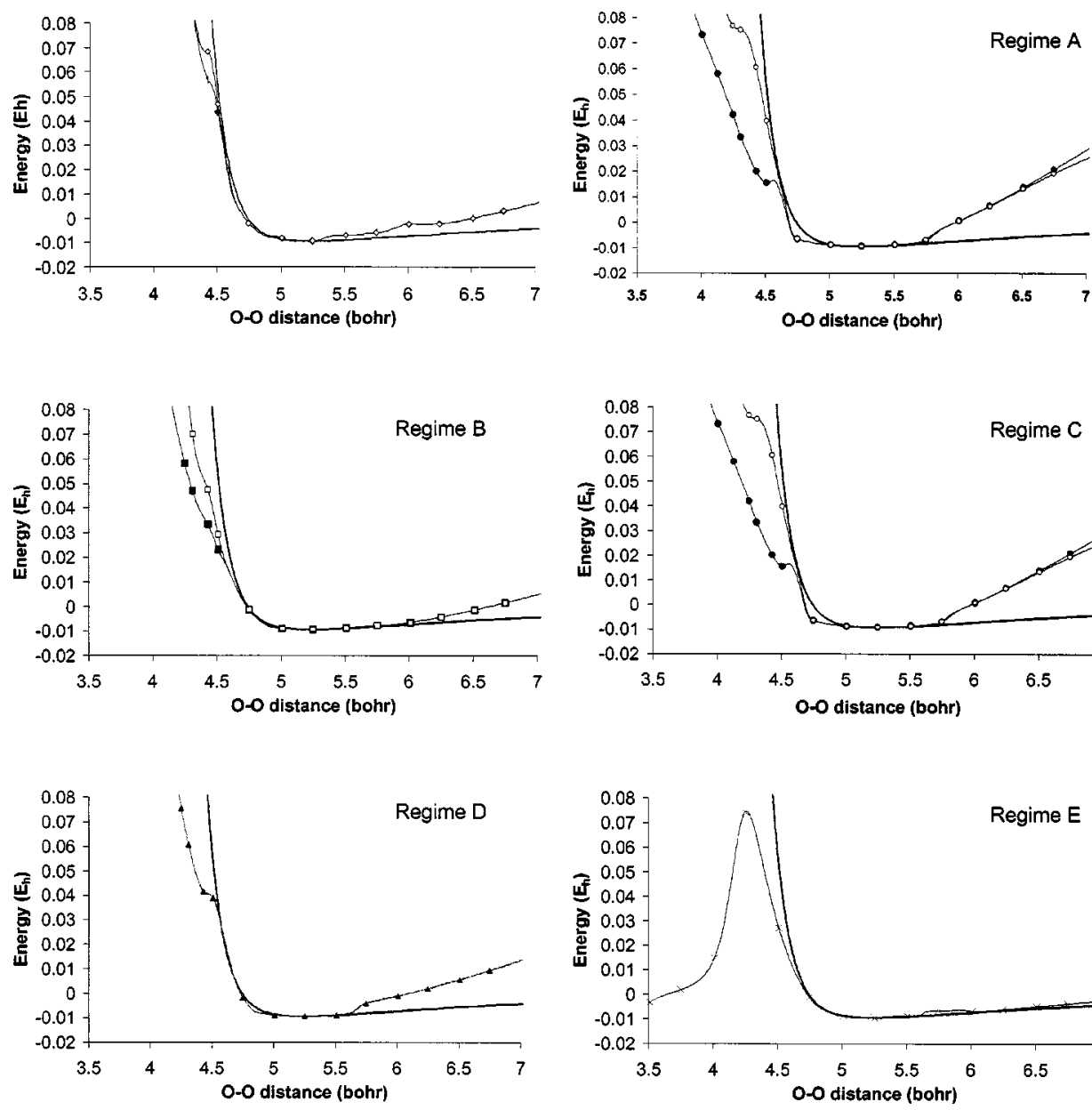
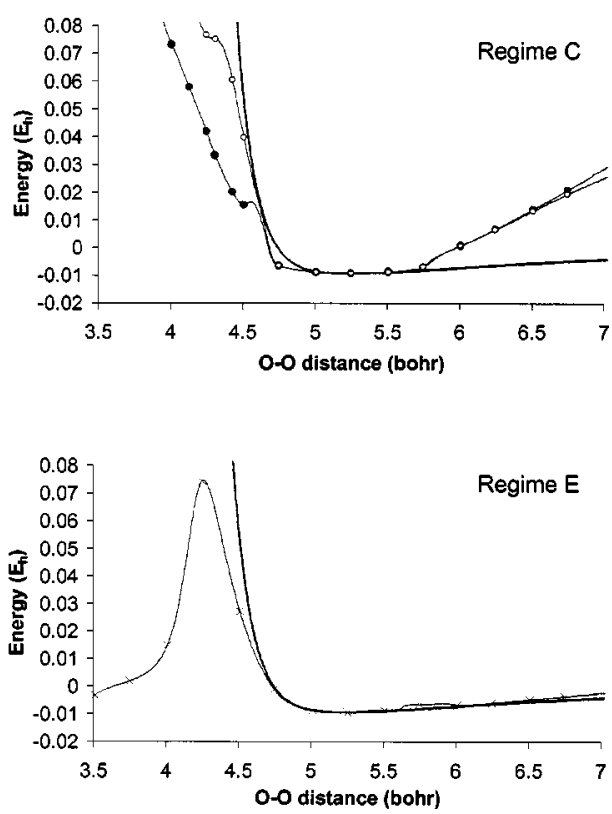

FIG. 4. PES profiles along the $\mathrm{O}-\mathrm{O}$ stretching normal mode displacement vector for the exact SW91 PES (solid line) and the one-point interpolated surface (dashed line), the regime (A) PES (diamonds), the regime (B) PES (squares), the regime (C) PES (circles), the regime (D) PES (triangles), and the regime (E) PES $(\times)$. All surfaces except the exact and one point are described by data sets containing 100 data points. Open symbols indicate the use of a $0.1 E_{h}$ energy screening cutoff, shaded symbols indicate use of a $1.0 E_{h}$ energy screening cutoff. 
TABLE III. The vibrationally averaged O-O distance $(\AA)$ in the water dimer ground state calculated from the regime (B) DMC-sampled PES and the regime (E) trajectory-sampled PES. ${ }^{\mathrm{a}, \mathrm{b}}$

\begin{tabular}{ccc}
\hline \hline Number of PES data points & Vibrationally averaged O-O distance in the water dimer ground state \\
\hline SW91 PES & & \\
1 & $2.834 \pm 0.009$ & Trajectory sampling \\
& Regime (E) \\
100 & Degime (B) & \\
200 & $2.768 \pm 0.011$ & $2.802 \pm 0.007$ \\
300 & $2.786 \pm 0.007$ & $2.790 \pm 0.006$ \\
500 & $2.789 \pm 0.011$ & $2.793 \pm 0.009$ \\
\hline \hline
\end{tabular}

${ }^{\mathrm{a}}$ Uncertainties representing two standard errors of the mean, as calculated from 10 independent DMC simulations.

${ }^{b}$ Where the PES interpolation scheme introduced spurious wells in the PES (see text) resulting in a negative ZPE, the table entry has been omitted.

and $9 h$-weight points per sampling run. This scheme is more efficient than regimes $(\mathrm{A})$ and $(\mathrm{C})$ as it enables the addition of 10 PES data points per iteration, thus reducing the number of DMC-sampling runs required and significantly reducing the overall computational expense of the PES iteration procedure.

In summary, we have established that the most efficient method considered here for growing a PES for the DMC calculation of the fully anharmonic water dimer ZPE requires the following:

(a) use of the two-part weight function,

(b) DMC sampling,

(c) the addition of $1 \mathrm{rms}$ and $9 h$-weight points per DMCsampling run.

The convergence of the ZPE was found to be relatively insensitive to the energy screening threshold, although profiles of the potential energy surfaces obtained using a $0.1 E_{h}$ screening threshold were slightly more accurate than those obtained using the $1.0 E_{h}$ screening threshold. The use of an energy screening threshold during the PES growing process may therefore improve the efficiency of the process by preventing the addition of data points in the high energy regions of configuration space, which are less chemically important.

Having established regime (B) as the most efficient method for constructing the PES, convergence of the surface grown using this regime was then tested with respect to the calculation of the nuclear vibrational wave function and ground state properties. The convergence of the trajectorysampled surface [regime (E)] with respect to the wave function and ground state properties was also tested for comparison.

\section{Ground state properties}

The ground state nuclear vibrational wave function and vibrationally averaged $\mathrm{O}-\mathrm{O}$ distance were calculated from the regime (B) two-part weight potential energy surfaces at $10,25,50,75$, and 100 data points using a $0.1 E_{h}$ energy screening threshold. The $\mathrm{O}-\mathrm{O}$ distance was chosen for examination because this quantity has been indirectly measured experimentally. ${ }^{50}$ As the $\mathrm{O}-\mathrm{O}$ average bond length had not converged by 100 data points, the PES was grown further to 500 data points. Convergence runs were carried out at 200, 300, 400, and 500 data points. For comparison, the trajectory-sampled surface [regime $(\mathrm{E})$ ] was also grown to 500 data points, with convergence runs also carried out at 200, 300, 400, and 500 data points. The vibrationally averaged $\mathrm{O}-\mathrm{O}$ distances are reported in Table III, and this data is depicted graphically in Fig. 5. The $\mathrm{O}-\mathrm{O}$ distances from the 100 and 200 data point regime (E) surfaces have been omitted from Table III because these surface possessed spurious wells, as discussed above. The $\mathrm{O}-\mathrm{O}$ radial distribution func-

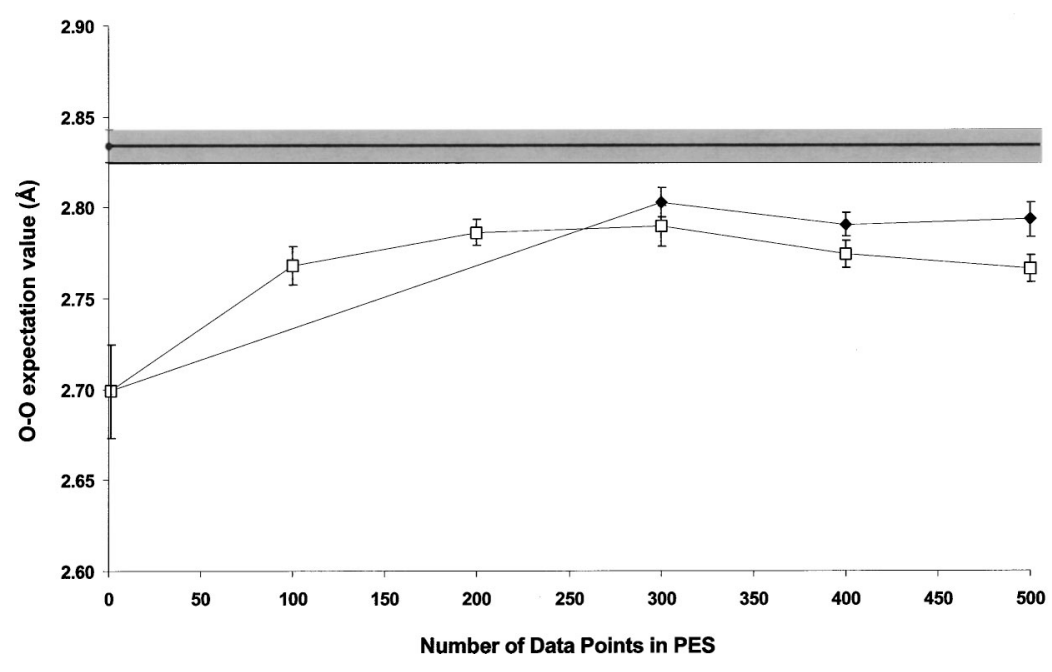

FIG. 5. Vibrationally averaged $\mathrm{O}-\mathrm{O}$ distances as a function of number of points in the PES data set for surfaces grown using regimes $(\mathrm{B})(\square)$ and $(\mathrm{E})(\bullet)$. 


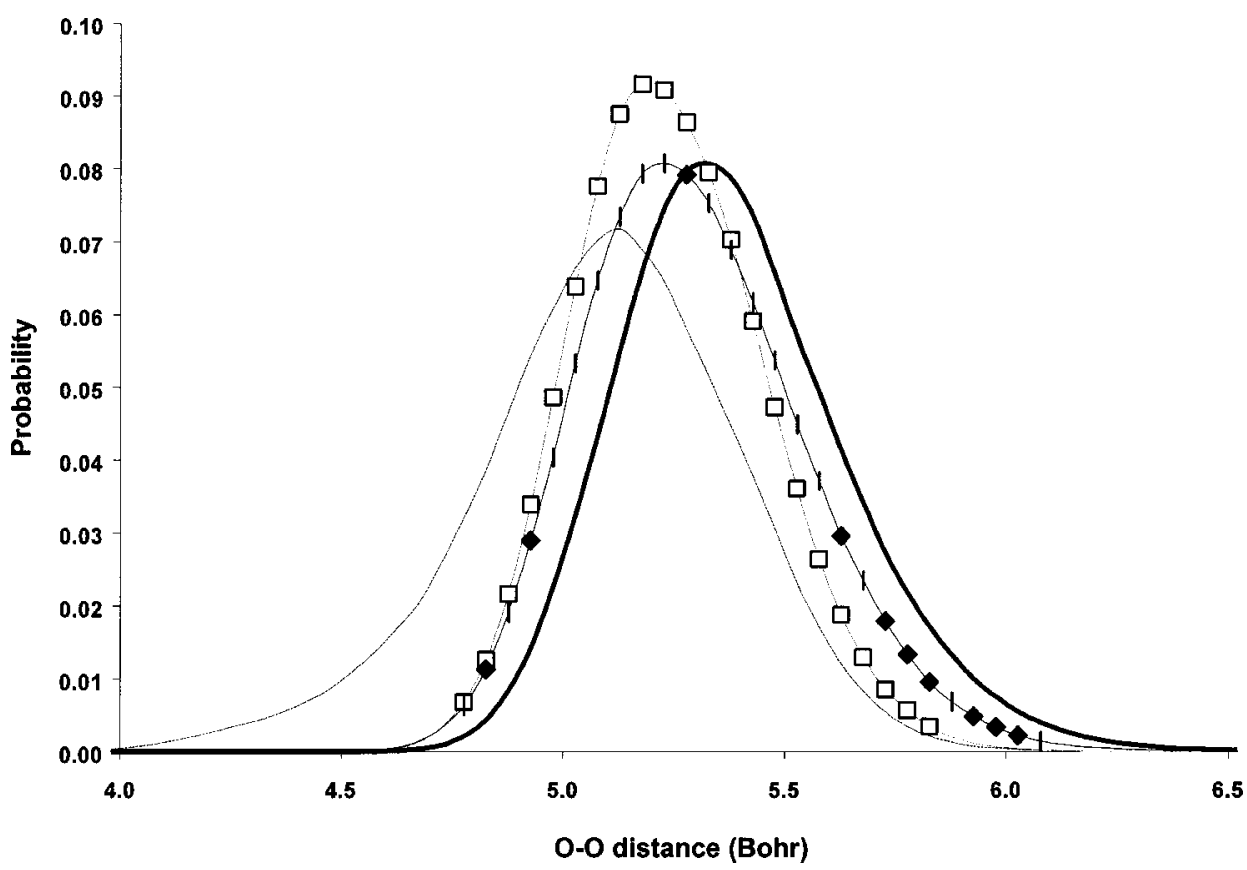

FIG. 6. The $\mathrm{O}-\mathrm{O}$ radial distribution function from the ground state wave function of the water dimer as calculated from the SW91 PES (solid line), the one-point interpolated surface (dashed line), and 500 point regime (B) $(\square)$ and regime (E) $(\downarrow)$ surfaces.

tions for the analytic, 1 point, 500 point regime (B) and 500 point regime $(\mathrm{E})$ surfaces are illustrated in Fig. 6. The $\mathrm{O}-\mathrm{O}$ radial distribution function represents the projection of $\left(|\psi| / 4 \pi r^{2}\right)^{2}$ onto the $\mathrm{O}-\mathrm{O}$ distance. The wave function histogram is projected onto the $\mathrm{O}-\mathrm{O}$ distance and then scaled by $4 \pi r^{2}$ prior to squaring the wave function amplitude in order to account for the Jacobian of the transformation from space fixed to internal coordinates. Profiles of the PES as a function of the $\mathrm{O}-\mathrm{O}$ stretching normal mode displacement vector are illustrated in Fig. 7 for the analytic, 1 point, 500 point regime (B) and 500 point regime (E) surfaces.

From Table III and Fig. 5 it is observed that convergence of the vibrationally averaged $\mathrm{O}-\mathrm{O}$ bond length is slow. From Fig. 6, it is observed that the shape of $\mathrm{O}-\mathrm{O}$ radial distribu- tion function also converges slowly, as the $\mathrm{O}-\mathrm{O}$ radial distribution functions from the interpolated surfaces have not converged to the analytic result even after addition of 500 data points. Despite the addition of 500 data points to the PES data set, the DMC-sampled surface underestimates the vibrationally averaged $\mathrm{O}-\mathrm{O}$ distance by approximately 0.068 $\AA$ and the trajectory-sampled PES underestimates it by 0.041 $\AA$. Initially, for the one point PES, the $\mathrm{O}-\mathrm{O}$ radial distribution function is both shifted to shorter $\mathrm{O}-\mathrm{O}$ distance and asymmetrically broadened, particularly at short $\mathrm{O}-\mathrm{O}$ distances, with respect to the radial distribution function calculated on the analytic SW91 PES. This occurs as a result of the predicted potential energy being significantly lower than the exact value at short $\mathrm{O}-\mathrm{O}$ distance and slightly higher

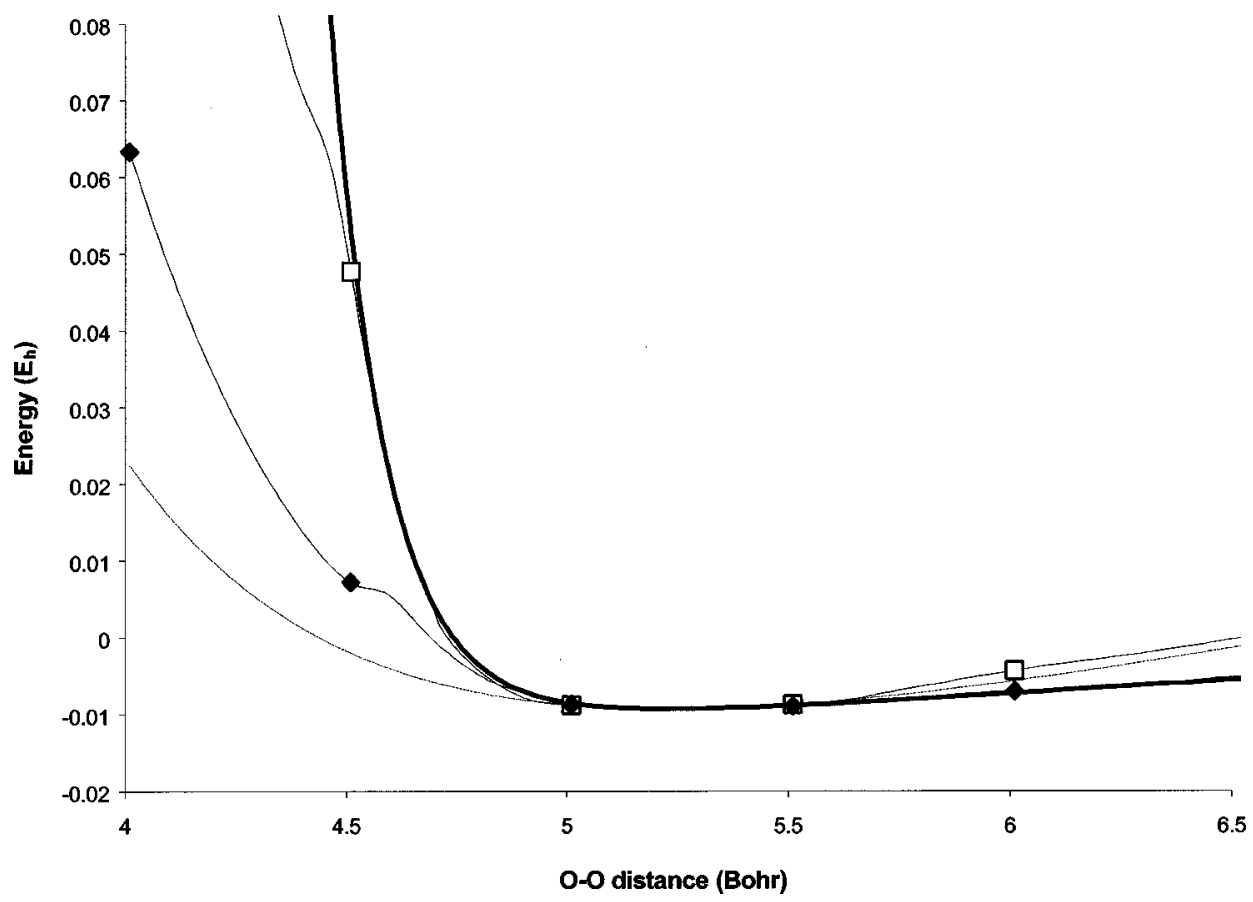

FIG. 7. PES profiles along the $\mathrm{O}-\mathrm{O}$ stretching normal mode displacement vector for the exact SW91 PES (solid line), the one-point interpolated surface (dashed line), and 500 point regime (B) $(\square)$ and regime (E) ( ) surfaces. 
than the exact value at long $\mathrm{O}-\mathrm{O}$ distance, as observed in Fig. 7. For both the DMC- and trajectory sampled surfaces, the steep repulsive wall at short $\mathrm{O}-\mathrm{O}$ distance becomes more accurately described upon addition of further data points. As a consequence, the radial distribution function narrows and shifts to longer $\mathrm{O}-\mathrm{O}$ distance. Figure 7 indicates that the short-range repulsive wall in the $\mathrm{O}-\mathrm{O}$ degree of freedom is more efficiently described by DMC sampling [regime (B)] than trajectory sampling [regime (E)]. Figure 7 also indicates that inaccuracies in the predicted potential energy at long $\mathrm{O}-\mathrm{O}$ distance were corrected by trajectory sampling but not by DMC sampling. Together, these inaccuracies were responsible for the shorter vibrationally averaged $\mathrm{O}-\mathrm{O}$ distances and the shift in the $\mathrm{O}-\mathrm{O}$ radial distribution function to shorter distance relative to the analytic SW91 PES for both the trajectory-sampled and DMC-sampled surfaces. Results at 500 data points were slightly better for the trajectorysampled PES than the DMC-sampled PES because trajectory sampling was more likely to place new data points at large $\mathrm{O}-\mathrm{O}$ distance. In terms of convergence of ground state properties, PES growing regimes incorporating both DMC and trajectory sampling may be more efficient than either method alone.

In general, because the shape of the wave function is strongly correlated to the topology of the PES, the quality of the wave function obtained from a PES is strongly correlated with the quality of the PES. One-dimensional slices through the wave function, such as the $\mathrm{O}-\mathrm{O}$ radial distribution function, therefore provide a sensitive measure of the convergence of the PES.

\section{SUMMARY}

The ZPE and ground state properties of the 12dimensional water dimer have been studied using DMC techniques on the SW91 analytic PES and interpolated potential energy surfaces derived from this analytic PES. The nature of the interpolating function was investigated by using either one- or two-part weight functions [Eqs. (4) and (5), respectively]. The iterative improvement of a PES, comprising initially only the global minimum configuration and its symmetry equivalents, was investigated using either DMC or trajectory simulations to sample configuration space along with a number of regimes for choosing which data points to add at each iteration.

In accordance with other published results on reactive systems, the two-part weight function was found to provide a significantly better description of the PES than the one-part weight function. Surfaces described using the two-part weight function exhibited significantly better convergence with respect to the ZPE.

The most efficient sampling regime considered here involved adding a single rms data point and $9 h$-weight points from a collection of geometries generated by DMC sampling of configuration space. This scheme was computationally efficient, as it decreased the number of DMC runs required per PES data point, hence decreasing the overall computational expense. This regime yielded a PES that was converged with respect to the ZPE upon addition of 50 data points to the PES data set. The vibrationally averaged $\mathrm{O}-\mathrm{O}$ distance and the
$\mathrm{O}-\mathrm{O}$ radial distribution function were more sensitive to the topology of the PES, requiring the addition of more than 500 data points to achieve convergence.

All the DMC based sampling regimes were found to be more efficient than the trajectory-based-sampling regime for convergence of the zero-point energy. However, the investigation of trajectory-based regimes was limited to the method previously found to be most efficient for reactive systemstrajectory sampling in combination with one rms and one $h$-weight point chosen per iteration. The convergence of ground state properties, such as the vibrationally averaged $\mathrm{O}-\mathrm{O}$ distance or the $\mathrm{O}-\mathrm{O}$ radial distribution function may well be most efficiently achieved with a combination of DMC and trajectory-based sampling.

The use of an energy screening threshold was found to be useful in converging PES profiles in the $\mathrm{O}-\mathrm{O}$ degree of freedom and, by implication, in converging the PES itself. The screening threshold served to prevent the addition of data points in less chemically relevant regions of configuration space.

In conclusion, we find that PES's constructed using weighted Taylor series expansions in inverse bond length coordinates provided a means of generating PES's suitable for the calculation of ZPE and ground state properties of the loosely bound water dimer complex. The methods we have identified to construct the 12-dimensional water dimer PES should also be applicable both to other loosely bound complexes, as well as tightly bound molecular species. When combined with high quality ab initio calculations, these methods should be able to accurately characterize the PES of such species.

\section{ACKNOWLEDGMENTS}

D.L.C. would like to acknowledge the financial support of an Australian Postgraduate Research Award. This work has also been supported by Large Grant No. A00104447 from the Australian Research Council and by grants of computer time from the Australian Partnership in Advanced Computing (APAC) National Merit Allocation Scheme.

${ }^{1}$ J. E. Del Bene and M. J. T. Jordan, Int. Rev. Phys. Chem. 18, 119 (1999).

${ }^{2}$ J. E. Del Bene and M. J. T. Jordan, J. Am. Chem. Soc. 122, 4794 (2000).

${ }^{3}$ J. B. Anderson, J. Chem. Phys. 63, 1499 (1975).

${ }^{4}$ J. B. Anderson, J. Chem. Phys. 65, 4121 (1976).

${ }^{5}$ J. B. Anderson, J. Chem. Phys. 73, 3897 (1980).

${ }^{6}$ J. B. Anderson and B. H. Freihaut, J. Comput. Phys. 31, 425 (1979).

${ }^{7}$ D. F. Coker and R. O. Watts, Mol. Phys. 58, 1113 (1986).

${ }^{8}$ M. A. Suhm and R. O. Watts, Phys. Rep. 204, 293 (1991).

${ }^{9}$ J. K. Gregory and D. C. Clary, Chem. Phys. Lett. 228, 547 (1994).

${ }^{10}$ J. K. Gregory and D. C. Clary, Chem. Phys. Lett. 237, 39 (1995).

${ }^{11}$ J. K. Gregory and D. C. Clary, J. Chem. Phys. 102, 7817 (1995).

${ }^{12}$ J. K. Gregory and D. C. Clary, J. Chem. Phys. 103, 8924 (1995).

${ }^{13}$ J. K. Gregory and D. C. Clary, J. Phys. Chem. A 101, 6813 (1997).

${ }^{14}$ D. M. Benoit and D. C. Clary, J. Chem. Phys. 113, 5193 (2000).

${ }^{15}$ R. E. Miller, D. F. Coker, and R. O. Watts, J. Chem. Phys. 82, 3554 (1985).

${ }^{16}$ D. F. Coker and R. O. Watts, J. Phys. Chem. 91, 2513 (1987)

${ }^{17}$ M. Quack and M. A. Suhm, J. Chem. Phys. 95, 28 (1991).

${ }^{18}$ M. Quack, J. Stohner, and M. A. Suhm, J. Mol. Struct. 294, 33 (1993).

${ }^{19}$ B. M. Auer and A. B. McCoy, J. Phys. Chem. A 107, 4 (2003).

${ }^{20}$ A. W. Meredith and A. J. Stone, J. Phys. Chem. A 102, 434 (1998). 
${ }^{21}$ M. Lewerenz, J. Chem. Phys. 104, 1028 (1996).

${ }^{22}$ P. Niyaz, Z. Bacic, J. W. Moskowitz, and K. E. Schmidt, Chem. Phys. Lett. 252, 23 (1996).

${ }^{23}$ P. Sandler, J. O. Jung, M. M. Szczniak, and V. J. Buch, Chem. Phys. 101, 1378 (1994).

${ }^{24}$ D. M. Benoit and D. C. Clary, J. Phys. Chem. A 104, 5590 (2000).

${ }^{25}$ M. J. T. Jordan, K. C. Thompson, and M. A. Collins, J. Chem. Phys. 102, 5647 (1995).

${ }^{26}$ K. C. Thompson, M. J. T. Jordan, and M. A. Collins, J. Chem. Phys. 108, 8302 (1998)

${ }^{27}$ R. P. A. Bettens and M. A. Collins, J. Chem. Phys. 111, 816 (1999).

${ }^{28}$ M. A. Collins, Theor. Chem. Acc. 108, 313 (2002).

${ }^{29}$ R. P. A. Bettens, J. Am. Chem. Soc. 125, 584 (2003).

${ }^{30}$ C. Leforestier, F. Gatti, R. S. Fellers, and R. J. Saykally, J. Chem. Phys. 117, 8710 (2002).

${ }^{31}$ O. Matsuoka, E. Clementi, and M. Yoshimine, J. Chem. Phys. 64, 1351 (1976).

${ }^{32}$ J. R. Reimers, R. O. Watts, and M. L. Klein, Chem. Phys. 64, 95 (1982).

${ }^{33}$ W. L. Jorgensen, J. Chandrasekhar, J. D. Madura, R. W. Impey, and M. L.

Klein, J. Chem. Phys. 79, 926 (1983).

${ }^{34}$ R. O. Watts, Chem. Phys. 26, 367 (1977).

${ }^{35}$ S. C. Althorpe and D. C. Clary, J. Chem. Phys. 101, 3603 (1994).
${ }^{36}$ M. J. T. Jordan, K. C. Thompson, and M. A. Collins, J. Chem. Phys. 103, 9669 (1995).

${ }^{37}$ M. Brouard, I. Burak, D. Minayev et al., J. Chem. Phys. 118, 1162 (2003).

${ }^{38}$ D. H. Zhang, M. A. Collins, and S.-Y. Lee, Science 290, 961 (2000).

${ }^{39}$ M. Yang, D. H. Zhang, M. A. Collins, and S.-Y. Lee, J. Chem. Phys. 114, 4759 (2001)

${ }^{40}$ R. P. A. Bettens, M. A. Collins, M. J. T. Jordan, and D. H. J. Zhang, J. Chem. Phys. 112, 10162 (2000).

${ }^{41}$ G. E. Moyano and M. A. Collins, J. Chem. Phys. 119, 5510 (2003).

${ }^{42}$ M. A. Collins and L. Radom, J. Chem. Phys. 118, 6222 (2003).

${ }^{43}$ R. O. Fuller, R. P. A. Bettens, and M. A. Collins, J. Chem. Phys. 114, 10711 (2001)

${ }^{44}$ M. A. Collins, S. Petrie, A. J. Chalk, and L. J. Radom, J. Chem. Phys. 112, 6625 (2000)

${ }^{45}$ R. P. A. Bettens, T. A. Hansen, and M. A. Collins, J. Chem. Phys. 111, 6322 (1999)

${ }^{46}$ M. A. Collins and R. P. A. Bettens, J. Chem. Phys. 111, 816 (1999).

${ }^{47}$ R. P. A. Bettens and M. A. Collins, J. Chem. Phys. 109, 9728 (1998).

${ }^{48}$ R. P. A. Bettens and M. A. Collins, J. Chem. Phys. 108, 2424 (1998).

${ }^{49}$ K. C. Thompson and M. A. Collins, J. Chem. Soc., Faraday Trans. 93, 871 (1997).

${ }^{50}$ J. A. Odutula and T. R. Dyke, J. Chem. Phys. 72, 5062 (1980). 